ORIGINAL RESEARCH PAPER

\section{CHOROIDAL VASCULARITY INDICES AND COEXISTING MORPHOLOGICAL CHANGES IN POLYPOIDAL CHOROIDAL VASCULOPATHY. A COMPARATIVE ANALYSIS BETWEEN TWO TREATMENT STRATEGIES}

Ophthalmology

KEY WORDS: Choroidal

Vascularity Index, Polypoidal Choroidal Vasculopathy,

Enhanced Depth Imaging, Image Binarization

\title{
Divya Alex*
}

MS, FICO, FVRS Department of vitreoretinal services, Giridhar Eye Institute, Ponneth Temple Road, Kadavanthra, Cochin 682020, Kerala, India.

*Corresponding Author

Purpose: Compare the effect of Combination therapy (PDT+Anti-VEGF) and Anti-VEGF monotherapy on choroidal vascularity indices and morphological parameters in Polypoidal ChoroidalVasculopathy (PCV).

Methods: Retrospective, cohort study involving 33eyes with a diagnosis of PCV and had visible sclerochoroidal boundary on enhanced depth imaging. Cases were treated either with combination $(n=17)$ or Anti-VEGF monotherapy $(n=16)$. Demographic details, visual acuity assessment, OCT analysis was considered from baseline to the 3rd and 6th month follow-up visits. Choroidal vascularity analysis including choroidal thickness, Total Choroidal Surface Area

터. (TCSA), Total Stromal area (TSA), Total Luminal Area (TLA) and Choroidal Vascularity Index (CVI) assessment were done with ImageJ software using the technique of image binarization.

Results: Disease activity was significantly higher in the Anti-VEGF monotherapy arm compared to the combination therapy arm both at 3 and 6 months. When compared to baseline values, there was statistically significant decrease in choroidal thickness, Double layer sign (DLS) width, TCSA, TSA and TLA $(P<0.05)$ in the combination therapy arm. Whereas, Anti-VEGF monotherapy arm showed an increase in the mean sub foveal choroidal thickness and DLS width at both visits. Complete collapse of PED, reduction in DLS width which was achieved only in combination therapy arm showed significant positive correlation with the resolution of the disease. CVI did not show a statistically significant reduction in both the arms.

Conclusion: In view of better outer retinal and choroidal morphological changes and vascular remodelling, our study strongly supports the superiority of combination therapy over Anti-VEGF monotherapy in PCV.

\section{INTRODUCTION}

Choroid, the vascular coating of the eye plays a predominant role in the ocular health. Choroid has the highest blood flow of any tissue in the body ${ }^{[1]}$ and has been implicated in the pathogenesis of many intraocular diseases such as agerelated macular degeneration, myopic macular degeneration and especially pachychoroid disease spectrum including Polypoidal Choroidal Vasculopathy (PCV).$^{[2-5]}$

Unlike retina which has a consistent pattern, evaluating the morphometry of choroid is challenging because of its complex and variable architecture. Morphological and vascular analyses of the choroid have been revolutionised with the advent of enhanced depth imaging (EDI) Optical coherence tomography (OCT). Sonoda et al first described a method for computing luminal and interstitial areas in the choroid as a means to quantify vascular status of the choroid by image binarization. ${ }^{[6]}$ Recently, Choroidal Vascularity Index (CVI); (ratio of luminal area to total choroidal area) was introduced as an OCT marker to assess the vascularity of sub foveal choroid by Agarwal et al. ${ }^{[7]}$ CVI has been availed as a supportive tool in monitoring disease progression in diseases like VKH and Stargardts. ${ }^{[8-9]} \mathrm{A}$ decrease in CVI was associated with a decrease in visual function in eyes with Stargardts disease. In VKH patients, it has been shown that with treatment there is significant reduction in CVI over time.CVI appears to be a more robust tool compared with SFCT for choroidal changes. However, the understanding of CVI in PCV is limited.

Available treatment modalities for PCV include verteporfin photodynamic therapy (PDT), Anti-VEGF therapy and thermal laser photocoagulation. PDT is thought to induce constriction of the choroidal vessels leading to hypoxia in both the choroid and retinal pigment epithelium, which causes upregulation of VEGF. ${ }^{[10-12]}$ Combination therapy comprising PDT and Anti-VEGF will inhibit VEGF expression, increase the efficacy of both PDT and pharmacologic agents. Anti-VEGF also protect against choroidal ischemia and retinal pigment epithelium damage caused by PDT as well as result in resolution of exudative changes in the retina. ${ }^{[11-12]}$ The three landmark trials EVEREST, ${ }^{[13]}$ LAPTOP $^{[14]}$ and FUJISAN ${ }^{[15]}$ study established the role of combination therapy of full-fluence PDT (greater polyp closure rate) with anti-VEGF agents (better visual outcomes) in initiating therapy for this disease entity.

Sonoda etal by using the technique of binarization have proven that the choroidal remodelling after PDT is mainly due to a decrease in the luminal areas. ${ }^{[6]}$ But the vascular remodelling after the antiVEGF therapy is poorly understood. The answer to the better outcomes with combination therapy rather than with Anti-VEGF monotherapy should lie within the choroid. Our study aimed to elucidate the reason for the same. The purpose of the current study was to compare the effect of Combination therapy (PDT+Anti-VEGF) and Anti-VEGF monotherapy on choroidal vascular remodelling and retinal morphometry in PCV.

\section{MATERIALS AND METHODS:}

We retrospectively analysed the electronic medical records of patients diagnosed with PCV who presented to our institute between June 2014 and March 2018. Approval from the ethics committee review board was obtained. The research followed the ethical standards as laid down in the 1964 Declaration of Helsinki and its later amendments.

\section{Inclusion criteria}

Treatment naive PCV cases with a minimum 6 month follow up after initial treatment and eyes with visible choroidal outer boundaries on spectral domain OCT (SD-OCT) were included in the study. Diagnosis and case selection of PCV was made purely based on Everest criteria; Presence of early subretinal nodular hyper fluorescence appearing within the first $5 \mathrm{~min}$ of ICG dye injection and at least one of the following diagnostic criteria: (1)Nodular appearance of the polyp (2)Hypo fluorescent halo around the nodule (3)Abnormal vascular channel(s) supplying the polyps (4)Pulsatile filling of polyps (5)Orange subretinal nodules corresponding to the hyperfluorescent area on ICGA.

\section{Exclusion criteria}

1)Coexisting macular/ vitreoretinal pathology 2) history of any vitreous surgery 3 ) history of previous treatment in the 
diseased eye in form of laser/ Anti-VEGF therapy 4) recent ocular inflammation 5) pregnancy 6) massive sub macular haemorrhage or exudation which obscured the choroidal vasculature in SD OCT 7) any participants with poor visibility of choroidal boundaries were excluded from the study.

Participants $(\mathrm{n}=33)$ were classified into two arms. Combination therapy arm $(n=17)$ who underwent Full fluence Photodynamic therapy (FFPDT) followed by loading dose (3 doses) of Anti-VEGF therapy. Anti-VEGF monotherapy arm $(\mathrm{n}=16)$ who received only Anti-VEGF injections 3 loading doses followed by pro re nata (PRN) regimen till 6 months. Disease activity at $3^{\text {rd }}$ month is defined as the presence of SRF/IRF even after 3 consecutive Anti-VEGF injections. Presence or reappearance of SRF/IRF at 6 months is considered as disease activity at 6 months. Complete resolution of SRF and IRF was considered as resolution for the study.

If eyes in combination therapy arm had recurrence of $\mathrm{SRF}$ or IRF between $3^{\text {rd }}$ and $6^{\text {th }}$ month, PRN regimen was followed. All the participants underwent a comprehensive ophthalmic examination including best-corrected visual acuity (BCVA), intraocular pressure measurement using Goldmann applanation tonometer, and dilated fundoscopic examination using $78 \mathrm{D}$ and $20 \mathrm{D}$.

\section{Choroidal Imaging}

As per defined protocol of the institution, Spectral domain optical coherence tomography (SD-OCT) was performed in each case using Heidelberg Spectralis HRA + OCT (Heidelberg Engineering, Germany) (1) macular dense scan (area $20^{\circ} * 20^{\circ}$, no of B scans: 49 , distance between B scans: 120 mm. (2) Enhanced depth imaging (EDI) at fovea. The EDI automatically sets the choroid closer to the zero-delay line and provides better visualization of the choroidal vasculature and sclerochoroidal interface. Choroidal thickness was measured using the inbuilt caliper as the distance from the outer portion of hyperreflective RPE to the inner portion of hyperreflective zone corresponding to the sclerochoroid junction. Width of the Double layer sign (DLS) (SDOCT finding defined as two hyperreflective lines corresponding to the separation of the Retinal pigment epithelium from the bruchs membrane) and PED height were analysed at each visit.

Image Binarization and Computation of Choroidal Vascularity Index

The EDI passing through the fovea was selected for image binarization. Central 3500microns (equidistant from fovea to both nasal and temporal sides) was segmented using the protocol described by Sonoda et al ${ }^{[7]}$ with few modifications described by Agarwal et al ${ }^{[8]}$. The image binarization and computation of CVI was done using Image s software (version 1.49; National Institutes of Health, Bethesda, USA). The subfoveal choroidal area with a width of $3.5 \mathrm{~mm}$, centred at the fovea, was selected and this constituted the region of interest. (Figurel) Intraclass correlation coefficient for our study was 0.89 for intrarater agreement and 0.86 for interrater agreement.

Figure 1: Process of binarization of choroidal vasculature (a) SDOCT EDI image through fovea. (b) marking the area of interest using polygon tool (c). 8bit converted autothreshold image (d) RGB color threshold image (e) final binarization into total luminal area(black) and total stromal area(yellow).

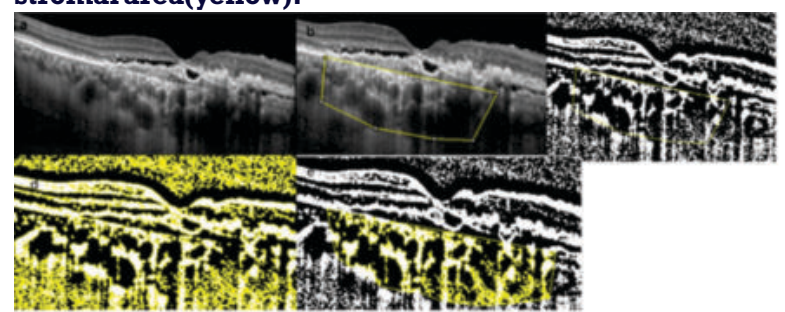

\section{RESULTS:}

Refining through the inclusion exclusion criteria, there were 33 participants, 17 in the combination arm and 16 in the Anti VEGF monotherapy arm. Both the group received 3 AntiVEGF injections in the first 3 months and from $3^{\text {rd }}$ to $6^{\text {th }}$ month, combination arm received an average of 1.25 injections and monotherapy arm received an average of 2.5 injections. At 3month follow-up, 13 (77.7\%) eyes in the combination therapy arm was inactive and whereas only $6(37.5 \%)$ eyes remained inactive in the Anti-VEGF monotherapy arm. At 6-month follow-up, $11(66.7 \%)$ eyes remained inactive in the combination group whereas only $5(31.2 \%)$ eyes remained inactive in the Anti-VEGF arm. On the contrary, more than 50\% of the eyes had still disease activity at 3 and 6 months in the monotherapy arm.

In the combination arm, there was a statistically significant improvement in BCVA $(P=0.01)$ at 3 rd and 6th month visits. The mean VA improved from Logmar 0.45(baseline) to 0.34 and to 0.31 at 3 and 6 months respectively. Whereas in monotherapy arm, the mean VA improved from Logmar 0.49 (baseline) to 0.44 and to 0.39 at 3 and 6 months respectively. There was a statistically significant improvement in BCVA $(\mathrm{P}=$ 0.04 ) only at the 6 th month visit in the monotherapy arm.

At $3^{\text {rd }}$ month, complete resolution of the subretinal fluid and intraretinal fluid was significantly higher in the combination arm $(P=0.003) .13(77.7 \%)$ eyes and $11(66.7 \%)$ eyes in combination arm remained fluid free at 3 - and 6 -month followup. Whereas at 3 months, $10(64.6 \%)$ eyes and at 6 months 11 $(68.7 \%)$ eyes in the monotherapy arm had subretinal or intraretinal fluid. Combination arm showed statistically significant decrease in choroidal thickness in EDI, DLS, TCSA, TSA \& TLA $(P=0.02)$ at 3- and 6-month follow-up visit. Monotherapy arm could reveal a statistically significant reduction only in the total luminal area $(\mathrm{P}=.039)$. However, there was no statistically significant difference in CVI ( $P=$ 0.65 ) at 3 and 6 month follow-up visit in both the arms.

In combination arm, the mean sub foveal choroidal thickness decreased from $376+83 u$ at baseline to $332+89 u(P=0.01)$ at 3 months and further $321+76 \mathrm{u}$ at 6 months. Complete collapse of PED was achieved in 12 (74\%) eyes. In monotherapy arm, the mean sub foveal choroidal thickness increased from $358+102 u$ at baseline to $361+97 u(P=0.04)$ at 3 months and further increase to $368+89 u(P=0.037)$ at 6 months. (Table 1\&2). In the combination therapy arm, the mean DLS width decreased from $2150+344 \mathrm{u}$ at baseline to $1860+278 \mathrm{u}(\mathrm{P}=0.03)$ at 3 months and further $1664 \mathrm{u}+276 \mathrm{u}$ at 6 months $(P=0.02)$. Monotherapy arm showed significant increase in the DLS width in 3rd and 6th month visit. The mean DLS width increased from $2630+454 \mathrm{u}$ at baseline to $2786+$ $478 \mathrm{u}$ at 3 months and further $2968 \mathrm{u}+276 \mathrm{u}(\mathrm{P}=0.001)$ at 6 months which showed a significant positive correlation with disease recurrence. Complete collapse of PED, reduction in DLS width which was achieved only in PDT arm showed positive correlation with the disease resolution.

Table 1: Baseline, $3^{\text {rd }}$ and $6^{\text {th }}$ month visit characteristics of PDT and Anti-VEGF groups.

\begin{tabular}{|l|l|l|l|l|l|l|}
\hline \multirow{4}{*}{} & \multicolumn{6}{|c|}{ Mean \pm SD (P) } \\
\cline { 2 - 7 } & \multicolumn{3}{|c|}{ PDT } & \multicolumn{3}{c|}{ ANTI-VEGF } \\
\cline { 2 - 7 } & Baseline & $\mathbf{3 ~ M}$ & $\mathbf{6 ~ M}$ & $\begin{array}{l}\text { Baseli } \\
\text { ne }\end{array}$ & $\mathbf{3 ~ M}$ & $\mathbf{6 ~ M}$ \\
\hline BCVA & $0.45 \pm$ & $0.34 \pm$ & $0.31 \pm$ & $0.49 \pm$ & $0.44 \pm$ & $0.39 \pm$ \\
& 0.299 & 0.358 & 0.293 & 0.281 & 0.351 & 0.333 \\
& & $(0.033 *$ & $(0.010 *$ & & $(0.389)$ & $(0.004 *$ \\
& & $*)$ & $*)$ & & & $*)$ \\
\hline CVI & $67.55 \pm$ & $68.77 \pm$ & $67.97 \pm$ & $68.90 \pm$ & $67.66 \pm$ & $68.49 \pm$ \\
& 4.755 & 3.396 & 3.413 & 4.918 & 2.809 & 3.026 \\
& & $(0.327)$ & $(0.560)$ & & $(0.649)$ & $(0.646)$ \\
\hline TCSA & 3654521. & 309253 & 312305 & 331123 & 354097 & 314285 \\
& $71 \pm$ & $4.25 \pm$ & $7.58 \pm$ & $9.61 \pm$ & $6.45 \pm$ & $4.13 \pm$ \\
& 1554771. & $127369)$ & $148675)$ & 108816 & 125289 & 117204 \\
\hline
\end{tabular}




\begin{tabular}{|c|c|c|c|c|c|c|}
\hline & 579 & $\begin{array}{l}3.665 \\
(0.025 * \\
*)\end{array}$ & $\begin{array}{l}7.960 \\
(0.019 * \\
*)\end{array}$ & 3.865 & $\begin{array}{l}8.299 \\
(0.078)\end{array}$ & $\begin{array}{l}2.467 \\
(0.066)\end{array}$ \\
\hline TLA & $\begin{array}{l}2426521 \\
13 \pm \\
922220.1 \\
26\end{array}$ & \begin{tabular}{|l|}
212808 \\
$7.70 \pm$ \\
891624. \\
348 \\
$(0.039 *$ \\
$*)$
\end{tabular} & \begin{tabular}{|l|}
212424 \\
$8.68 \pm$ \\
101603 \\
2.508 \\
$(0.028 *$ \\
$*)$
\end{tabular} & $\begin{array}{l}257849 \\
7.22 \pm \\
700417 \\
218\end{array}$ & \begin{tabular}{|l|}
280129 \\
$5.01 \pm$ \\
787432. \\
778 \\
$(0.079)$
\end{tabular} & \begin{tabular}{|l|}
243394 \\
$5.28 \pm$ \\
735574. \\
848 \\
$(0.039 *$ \\
$*)$
\end{tabular} \\
\hline \begin{tabular}{|l} 
DLS \\
Widt \\
h
\end{tabular} & $\begin{array}{l}2150.92(6 \\
30.791)\end{array}$ & $\begin{array}{l}1860.00 \\
(666.07 \\
1) \\
(<0.001 \\
* *)\end{array}$ & $\begin{array}{l}2067.69 \\
(722.79 \\
1) \\
(<0.001 \\
* *)\end{array}$ & \begin{tabular}{|l}
2630.38 \\
$(1162.6$ \\
$35)$
\end{tabular} & \begin{tabular}{|l|}
2786.75 \\
$(722.63$ \\
$5)$ \\
$(<0.001$ \\
$* *)$
\end{tabular} & $\begin{array}{l}2968.37 \\
(693.93 \\
2) \\
(<0.001 \\
* *)\end{array}$ \\
\hline
\end{tabular}

**- significant $\mathbf{P}$ value when compared to the baseline values.

Table2: Correlation of baseline parameters with disease activity of both the groups

\begin{tabular}{|l|c|c|c|c|}
\hline \multirow{2}{*}{$\begin{array}{l}\text { Disease activity } \\
\text { at months }\end{array}$} & \multicolumn{2}{|c|}{ PDT } & \multicolumn{2}{c|}{ ANTI-VEGF } \\
\cline { 2 - 5 } & $\begin{array}{l}\text { Correlatio } \\
\text { Coefficient }\end{array}$ & $\begin{array}{c}\text { P } \\
\text { Value }\end{array}$ & $\begin{array}{c}\text { Correlation } \\
\text { Coefficient }\end{array}$ & $\begin{array}{c}\text { P } \\
\text { Value }\end{array}$ \\
\hline Baseline EDI & 0.192 & 0.459 & -0.379 & 0.147 \\
\hline $\begin{array}{l}\text { Baseline DLS } \\
\text { WIDTH }\end{array}$ & 0.577 & $\begin{array}{c}0.039 * \\
*\end{array}$ & 0.516 & $0.028 * *$ \\
\hline Baseline TCSA & 0.481 & 0.051 & -0.379 & 0.147 \\
\hline Baseline TLA & 0.457 & 0.065 & -0.348 & 0.187 \\
\hline Baseline CVI & -0.241 & 0.352 & 0.095 & 0.727 \\
\hline
\end{tabular}

**- significant $\mathbf{P}$ value when compared to the baseline values.

\section{DISCUSSION:}

Segmentation of the choroid into different layers and its vascular components has been a significant challenge. The new automated segmentation software to binarize the choroidal structure has thrown light into the better understanding of the choroidal vasculature. It is a well-known fact that the recurrence rate of PCV with combination therapy is much lower than that of PCV treated with Anti-VEGF alone. [14-16] Studying the choroidal remodelling after both these therapies will give a better clue in explaining the reason for the same. Our present study found the changes in the choroidal morphology and vascularity after the two different established treatment modalities in PCV.

Histologically, the choroid is composed of blood vessels (total luminal area) and interstitial tissues (total stromal area). For a clinical approach, we compared the effect of combination and Anti-VEGF monotherapy on the luminal and interstitial areas of the choroid. Our results showed that both areas decreased in size, but the luminal area decreased more than the interstitial area with combination therapy. CVI did not show a statistical decrease; may be because of the proportional decrease in the luminal and interstitial area. Whereas the Anti VEGF arm showed a statistically significant decrease in the luminal area only at 6 months of follow-up. Anti VEGF arm did not show a statistically significant decrease in the total stromal area. Hence our study proves, combination therapy induces better choroidal vascular remodelling than Anti-VEGF monotherapy.

The mechanism of action of PDT is postulated to be short-term choriocapillaris hypoperfusion and long-term choroidal remodelling leading to reductions in choroidal congestion, vascular hyperpermeability and extravascular leakage. Schlötzer-Schrehardt etal detected choriocapillaris occlusion in the region of PDT application l week after standard PDT in human eyes by histopathological analysis. ${ }^{[1,18]}$ These all facts are supported by our results in the combination arm in view of statistically significant reduction in total luminal area, total stromal area and total choroidal surface area which occurred both at 3 and 6 months.

Monotherapy arm showed statistically significant reduction only in the total luminal area and was achieved only at the end of 6 months. Since there is a decline in the luminal areas, it can be assumed that either the number of vessels reduced or the diameter of vessels reduced. VEGF can dilate the vascular channels by stimulating endothelial nitric oxide synthase (eNOS) dependent pathways, ${ }^{[19]}$ and thus downregulation of VEGF with Anti-VEGF agents can substantiate the statistically significant reduction in total luminal area even in monotherapy arm. Vascular endothelial growth factor plays an important role in preserving the homeostasis of the choroid, and it is secreted mainly on the basal side of the retinal pigment epithelium. VEGF increases vascular permeability; this allows intravascular osmotically active molecules to move into the interstitial tissue, resulting in the interstitial tissue swelling. ${ }^{[20]}$ Thus, an inhibition of the release of VEGF might result in a decrease in the release of osmotically active molecules. So theoretically Anti-VEGF monotherapy can also cause reduction in the total luminal area. Our study showed consistent results with the same.

Another important finding in the study was the coexistent outer retinal morphological changes in the combination therapy arm. Complete collapse of the PED was attained in $64.7 \%$ of the combination arm versus $23.5 \%$ in the monotherapy arm. The same was associated with a strong positive correlation with the resolution of disease. Reduction in the DLS width which was attained only in the Combination arm also showed a significant positive correlation with disease resolution. Surprisingly, there was an increase in the DLS width in the monotherapy arm. Double layer sign is supposedly the encroaching type one choroidal neovascularization. Our study proves that Anti-VEGF monotherapy could not resist the growth of the choroidal neovascularisation in PCV, but it could help in reducing the exudation from the same. Whereas, the combination therapy using PDT could induce a reduction in the existing neovascular network as well as resist the further growth of the same. Hence our study proves, combination therapy induces better outer retinal remodelling than Anti-VEGF monotherapy in PCV which may be the reason for the less recurrence rate. This is in consistent with Shimuzu etal study which proved PDT causes microstructural outer retinal changes. ${ }^{[21]}$

We do acknowledge the potential limitations of this analysis including the retrospective nature and the relatively small sample size especially due to the poor visibility of the sclerochoroidal junction and lack of Aflibercept as a treatment option because of the extreme small cohort. Future studies, looking at a larger sample size may be definitely helpful to further elucidate the lacunae between the two treatment strategies.

\section{CONCLUSION:}

CVI could be a parameter demonstrating the choroidal vessel congestion and hyperpermeability, which is the primary pathogenic mechanism in PCV. Choroidal Vascular parameters could also be a useful to understand the effect of therapeutic intervention. Being primarily a type 1 choroidal neovascularization, administration of intravitreal anti-VEGF therapy should benefit in PCV. But PDT addresses the choroid, the origin and root cause of the disease activity in PCV. Our study in view of better outer retinal and choroidal morphological changes and vascular remodelling, strongly support the superiority of combination therapy over AntiVEGF monotherapy.

\section{REFERENCES}

1. Alm, A. \& Bill, A. Ocular and optic nerve blood flow at normal and increased intraocular pressures in monkeys (Macacairus): a study with radioactively labelled microspheres including flow determinations in brain and some other tissues. Exp Eye Res 1973; 15, 15-29

2. Spaide RF. Enhanced depth imaging optical coherence tomography of retinal pigment epithelial detachment in agerelated macular degeneration. Am J 
Ophthalmol.2009;147:644-652.

3. Zarbin MA. Current concepts in the pathogenesis of agerelated macular degeneration. Arch Ophthalmol. 2004;122:598-614

4. McLeod DS, Grebe R, Bhutto I, Merges C, Baba T, LuttyGA.Relationship between RPE and choriocapillaris in age-related macular degeneration. Invest OphthalmolVis Sci. 2009;50:4982-4991

5. Uyama M, Wada M, Nagai Y, Matsubara T, Matsunaga H, Fukushima I, Takahashi K Polypoidal choroidal vasculopathy: natural history. Am J Ophthalmol,2002;133:639-648

6. Sonoda, S. et al. Luminal and stromal areas of choroid determined by binarization method of optical coherence tomographic images. Am J Ophthalmol159, 1123-1131,2015

7. Agrawal R, Gupta P, Tan KA, Cheung CM, Wong TY, Cheng CY. Choroidal vascularity index as a measure of vascular status of the choroid: Measurements in healthy eyes from a population-based study. Scientific reports. 2016 Feb 12;6:21090.

8. Agrawal, R., Li, L. K. H., Nakhate, V., Khandelwal, N., \& Mahendradas, P. (2016). Choroidal vascularity index in Vogt-Koyanagi-Harada disease: an EDI-OCT derived tool for monitoring disease progression. Translational vision science \& technology, 5(4), 7-7.

9. Ratra, D., Tan, R., Jaishankar, D., Khandelwal, N., Gupta, A., Chhablani, J. and Agrawal, R., 2018. Choroidal structural changes and vascularity index in stargardt disease on swept source optical coherence tomography. Retina, 38(12), pp.2395-2400.

10. Kiss CG, Simader C,Michels S, Schmidt-Erfurth U. Combination of verteporfin photodynamic therapy and ranibizumab:effects on retinal anatomy, choroidal perfusion and visual function in the protect study. Br J Ophthalmol 2008;92(12):1620-1627

11. Kaiser PK; Registry of Visudyne AMD Therapy Writing Committee. Verteporfin photodynamic therapy combined with intravitreal bevacizumab for neovascular age-related macular degeneration. Ophthalmology 2009;116(4):747-755,755.el.

12. Saito M, Shiragami C, Shiraga F, et al. Combined intravitreal bevacizumab and photodynamic therapy for retinal angiomatous proliferation. Am J Ophthalmol 2008;146(6):935-941.el

13. Koh A, Lee WK, Chen LJ, Chen SJ, Hashad Y, Kim H, Lai TY, Pilz S, Ruamviboonsuk P, Tokaji E, Weisberger A. EVEREST study: efficacy and safety of verteporfin photodynamic therapy in combination with ranibizumab or alone versus ranibizumab monotherapy in patients with ranibizumab or alone versus ranibizumab monotherapy in patients with
symptomatic macular polypoidal choroidal vasculopathy. Retina. 2012 Sep $1 ; 32(8): 1453-64$.

14. Oishi A, Kojima H, Mandai M, Honda S, Matsuoka T, Oh H, Kita M, Nagai T, Fujihara M, Bessho N, Uenishi M. Comparison of the effect of ranibizumab and verteporfin for polypoidal choroidal vasculopathy: 12-month LAPTOP study results. American journal of ophthalmology.2013 Oct 1;156(4):644-51.

15. Gomi F, Oshima Y, Mori R, Kano M, Saito M, Yamashita A, Iwata E, Fujisan Study Group. Initial versus delayed photodynamic therapy in combination with ranibizumab for treatment of polypoidal choroidal vasculopathy: The Fujisan Study.Retina.2015 Aug 1;35(8):1569-76.

16. Anantharaman G, Sheth J, Bhende M, Narayanan R, Natarajan S, Rajendran A Manayath G, Sen P, Biswas R, Banker A, Gupta C. Polypoidal choroidal vasculopathy: Pearls in diagnosis and management. Indian journal of ophthalmology. 2018 Jul;66(7):896.

17. Blaauwgeers HG, Holtkamp GM, Rutten $\mathrm{H}$, et al. Polarized vascular endothelial growth factor secretion by human retinal pigment epithelium and localization of vascular endothelial growth factor receptors on the inner choriocapillaris. Evidence for a trophic paracrine relation. Am J Pathol. 1999; 155:421-428.

18. Chan WM, Lam DS, Lai TY, Tam BS, Liu DT, Chan CK. Choroidal vascular remodelling in central serous chorioretinopathy after indocyanine green guided photodynamic therapy with verteporfin: a novel treatment at the primary disease level. British Journal of Ophthalmology. 2003 Dec $1 ; 87(12): 1453-8$

19. Izumi T, Koizumi H, Maruko I, Takahashi Y, Sonoda S, Sakamoto T, Iida T. Structural analyses of choroid after half-dose verteporfin photodynamic therapy for central serous chorioretinopathy. British Journal of Ophthalmology.2017 Apr 1;101(4):433-7

20. Maruko I, Iida T, Oyamada $\mathrm{H}$, et al. Choroidal thickness changes after intravitreal ranibizumab and photodynamic therapy in recurrent polypoidal choroidal vasculopathy.Am J Ophthalmol 2013;156:548-556

21. Shimizu K, Hashimoto Y, Azuma K, Nomura Y, Obata R, Takahashi H, Yanagi Y. Changes of outer retinal microstructures after photodynamic therapy for chronic central serous chorioretinopathy. Clinicalophthalmology (Auckland,NZ).2017;11:1505-12 